\title{
Exosomes from breast cancer cells stimulate proliferation and inhibit apoptosis of $\mathrm{CD} 133^{+}$cancer cells in vitro
}

\author{
JIANHUA SHI*, YI REN*, LINLIN ZHEN and XIAOLAN QIU \\ Department of Breast and Thyroid Surgery, Huai'an First People's Hospital, \\ Nanjing Medical University, Huai'an, Jiangsu 223001, P.R. China
}

Received December 8, 2013; Accepted July 22, 2014

DOI: $10.3892 / \mathrm{mmr} .2014 .2749$

\begin{abstract}
Exosomes are a family of bioactive vesicles that are secreted from various types of cell, including tumor cells. Exosomes derived from breast cancer cells have been demonstrated to perform important functions in tumor progression in vitro and in vivo. However, few studies exist regarding the function of exosomes in $\mathrm{CD}_{133^{+}}$breast cancer cells. In the present study, exosomes from 4T1 mouse breast cancer cells and mouse mammary gland epithelial cells were purified. The exosome-associated markers CD63, CD9 and CD81 were detected, and the size distribution and $\zeta$ potential of the exosomes were determined. Exosome uptake by $\mathrm{CD}_{133^{+}}$and CD133-4T1 cells was confirmed by confocal microscopy. An ATPlite assay indicated that the proliferation of $\mathrm{CD}_{133^{+}}$cells was increased and the apoptosis was suppressed by exosomes from 4T1 cells. Collectively, the findings of the present study demonstrate a novel mechanism by which the action of exosomes on $\mathrm{CD}_{133}{ }^{+} 4 \mathrm{~T} 1$ cells may contribute to breast cancer progression.
\end{abstract}

\section{Introduction}

Exosomes are vesicles of 30-100 nm in length, that were first identified in the 1980s and are secreted by a wide range of mammalian cell types, including cancer cells (1). Studies have demonstrated that tumor-derived exosomes may produce a double-effect in tumor pathology. One study by Wolfers et al (2) demonstrated that tumor antigens can be transferred by exosomes from tumor cells to dendritic cells (DCs), and thus function in antigen cross-presentation. Like DC-derived exosomes, exosomes from tumor cells carry major histocompatibility complex molecules

Correspondence to: Professor Jianhua Shi, Department of Breast and Thyroid Surgery, Huai'an First People's Hospital, Nanjing Medical University, 6 Beijing West Road, Huai'an, Jiangsu 223001, P.R. China

E-mail: jianhuashi2013@126.com

*Contributed equally

Key words: exosomes, breast cancer, CD133 along with tumor-specific antigens expressed in the parental tumor cells, including melan-A/MART1, mesothelin (3), silv (2) and carcinoembryonic antigen (4), which can be recognized by $\mathrm{T}$ cells. Together, these studies suggest that tumor-derived exosomes may be a novel candidate for tumor vaccine development. By contrast, a large number of studies have indicated diverse immunosuppressive effects of exosomes from tumor cells, including suppression of effector $\mathrm{T}$ cell activity (5), induction of apoptosis in activated T cells (6) and natural killer cells (7), modulation of differentiation in myeloid cells (8), and functional enhancement of regulatory $\mathrm{T}$ cells.

CD133 is a marker of cancer stem cells and its expression is associated with poor prognosis and chemoresistance in several types of solid tumors, including breast cancer (9). However, there is little evidence of the effects of tumor-derived exosomes on the $\mathrm{CD}_{133^{+}}$stem cell-like tumor cells. The current study aimed to determine the effect of $4 \mathrm{~T} 1$ breast cancer cell line-derived exosomes on the proliferation and apoptosis of CD133+ $4 \mathrm{~T} 1$ cells.

\section{Materials and methods}

Cell culture. The 4T1 mouse mammary carcinoma cell line was obtained from the American Type Culture Collection (Manassas, VA, USA) and cultured in Dulbecco's modified Eagle's medium (DMEM) supplemented with $10 \%$ fetal bovine serum (FBS) plus $100 \mathrm{U} / \mathrm{ml}$ penicillin and $100 \mu \mathrm{g} / \mathrm{ml}$ streptomycin. NMuMG mouse mammary gland epithelial cells (American Type Culture Collection) were cultured in DMEM supplemented with $10 \%$ FBS with $10 \mu \mathrm{g} / \mathrm{ml}$ insulin, $100 \mathrm{U} / \mathrm{ml}$ penicillin and $100 \mu \mathrm{g} / \mathrm{ml}$ streptomycin. All culture reagents were obtained from Invitrogen (Carlsbad, CA, USA).

For exosome isolation, 4T1 and NMuMG cells were cultured in medium with exosome-free FBS (exosomes in FBS were removed by ultracentrifugation at 100,000 $\mathrm{x}$ g overnight at $\left.4^{\circ} \mathrm{C}\right)$.

Exosome purification. Exosomes from 4T1 cells (4T1-Exo) or control exosomes from NMuMG cells (NMuMG-Exo) were isolated using a Total Exosome Isolation kit (\#4478359) purchased from Invitrogen. Briefly, culture media were collected and centrifuged at 2,000 x $\mathrm{g}$ for $30 \mathrm{~min}$ to remove cells and debris. The required volume of cell-free culture media was transferred to a fresh tube, and $0.5 \mathrm{X}$ volumes 
of the Total Exosome Isolation reagent were added prior to incubation at $4^{\circ} \mathrm{C}$ overnight. Following incubation, samples were centrifuged at $10,000 \times \mathrm{g}$ for $1 \mathrm{~h}$ at $4^{\circ} \mathrm{C}$. The supernatant was discarded and the pellets were resuspended in $1 \mathrm{X}$ phosphate-buffered saline (PBS) and stored at $-80^{\circ} \mathrm{C}$ until use.

Dynamic light scattering and $\zeta$ potential. Size distribution and $\zeta$ potential of exosomes were measured as described previously (10). In brief, exosomes were washed with double distilled (dd) $\mathrm{H}_{2} \mathrm{O}$ at $100,000 \mathrm{x} \mathrm{g}$ for $1 \mathrm{~h}$ and resuspended in $\mathrm{ddH}_{2} \mathrm{O}$ for analysis. Prior to measurement, samples were transferred to a cuvette (BrandTech Scientific, Inc., Essex, CT, USA) for dynamic light scattering analysis, or subjected to an electric field for $\zeta$ potential determination using the Zetasizer Nano ZS (Malvern Instruments, Ltd., Malvern, UK).

Transmission electron microscopy (TEM). For TEM analysis, exosomes were fixed with $2 \%$ paraformaldehyde in PBS; then they were dropped onto Formvar/Carbon on 200 mesh thick grids (Agar Scientific, Monterotondo, Italy) and left to dry at room temperature for $20 \mathrm{~min}$. Following a brief wash, the grids were fixed with $1 \% \mathrm{w} / \mathrm{v}$ glutaraldehyde in PBS, followed by several washing steps in distilled water. Samples were contrasted by $4 \%$ w/v Uranyl Acetate (Sigma-Aldrich, St Louis, MO, USA) and by UA-Methylcellulose mix solution (Sigma-Aldrich). The grid was dried at room temperature, viewed in a Tecnai 12 G2 Transmission Electron Microscope (FEI, Eindhoven, The Netherlands) and then analyzed with Olympus iTEM CE software (Olympus Soft Imaging Solutions $\mathrm{GmbH}$, Münster, Germany).

Western blot analysis. Exosomes were lysed with lysis buffer and boiled. Proteins were quantified by a Lowry assay using Bio-Rad Protein Assay kit I (Bio-Rad, Hercules, CA, USA). Lysates were subjected to SDS/PAGE (10\% gel) and the proteins were transferred on to a polyvinylidine fluoride membrane (Life Technologies, Grand Island, NY, USA). Subsequent to immunoblotting with antibodies, including rat anti-mouse CD9, goat anti-mouse CD63 and goat anti-mouse CD81 (Santa Cruz Biotechnology, Inc., Dallas, TX, USA), the reaction product was revealed with an Amersham ECL Western Blotting system (GE Healthcare Life Sciences, Chalfont, UK).

$\mathrm{CD} 133^{+}$cell sorting. To isolate the $\mathrm{CD}_{133^{+}}$and $\mathrm{CD} 133^{-}$fractions, 4T1 cells were resuspended in Hank's Balanced Salt Solution (HBSS; Gibco-BRL, Carlsbad, CA, USA) containing 2\% FBS and $10 \mathrm{mM}$ HEPES (Fisher Scientific, MA, USA ). The cell density was adjusted to $1 \times 10^{7} / \mathrm{ml}$ and the $4 \mathrm{~T} 1$ cells were stained with fluorescein isothiocyanate-conjugated human anti-mouse CD133 IgG antibody (\#130-105-226; Miltenyi Biotec, Inc., Cambridge, MA, USA). Subsequent to staining, the 4T1 cells were resuspended in HBSS containing $2 \%$ FBS and $1 \mathrm{mM}$ HEPES, filtered through a $40-\mu \mathrm{m}$ mesh filter, and the $\mathrm{CD}_{133^{+}}$and $\mathrm{CD} 133^{-}$4T1 fractions were sorted by a FACSAria cell sorter II (BD Biosciences, San Jose, CA, USA).

Exosome uptake assays. CD133+ and CD133- 4T1 cells were cultured in a 4 -well chamber for $24 \mathrm{~h}$, then the PKH26
(Sigma-Aldrich)-labeled NMuMG-Exo or 4T1-Exo were added into the wells and incubated with the cells for $12 \mathrm{~h}$ at $37^{\circ} \mathrm{C}$. The cells were then washed three times with cold PBS following incubation, fixed in $2 \%$ paraformaldehyde for $10 \mathrm{~min}$ at room temperature, permeabilized with $0.1 \%$ Triton $\mathrm{X}-100$ (Sigma-Aldrich) for $2 \mathrm{~min}$ at room temperature, washed three times with PBS and stained with DAPI (Life Technologies). Internalization of exosomes was observed under a A1R-A1 Confocal Microscope system (Nikon Corporation, Tokyo, Japan).

Proliferation assay. An ATPlite Luminescence Assay system (\#6016941; PerkinElmer, Inc., Waltham, MA, USA) was used to identify levels of proliferation in CD133+ and CD133-4T1 cells following incubation with the 4T1-Exo and NMuMG-Exo. Briefly, CD133+ ${ }^{+}$or CD133- $4 \mathrm{~T} 1$ cells $\left(5 \times 10^{4}\right)$ were cultured in 24 well plates for $24 \mathrm{~h}$ and then the 4T1-Exo and NMuMG-Exo $(10 \mu \mathrm{g})$ were respectively added and incubated for 24 or $48 \mathrm{~h}$. Mammalian cell lysis buffer $(50 \mu \mathrm{l})$ was added to each well and the wells were shaken in a orbital shaker at $250 \mathrm{x} \mathrm{g}$ for $5 \mathrm{~min}$. Then, $50 \mu \mathrm{l}$ substrate solution was added to the wells and the plate was shaken at $700 \mathrm{rpm}$ for another $5 \mathrm{~min}$. The plates were dark adapted for $10 \mathrm{~min}$ and the luminescence was measured.

Apoptosis assay. The 4T1 cells were treated with doxorubicin $(1 \mu \mathrm{M})$ to induce apoptosis, with or without $10 \mu \mathrm{g}$ NMuMG-Exo or 4T1-Exo for $12 \mathrm{~h}$, and then Annexin V/propidium iodide (PI) staining was performed to quantify the apoptosis levels in 4T1 cells. In brief, 4T1 cells were harvested and washed twice with PBS, and then resuspended in $1 \mathrm{X}$ binding buffer (BD Biosciences) at a concentration of $1 \times 10^{6}$ cells $/ \mathrm{ml}$. Cells were incubated with fluorescein isothiocyanate labeled anti-Annexin V antibody (5 $\mu \mathrm{l}$, eBioscience, San Diego, CA, USA) for $15 \mathrm{~min}$ at room temperature. After washing with binding buffer, the cells were resuspended in $200 \mu \mathrm{l}$ binding buffer. PI staining solution ( $5 \mu \mathrm{l}$, eBioscience) was added and the cells were analyzed using a BD Accuri C6 flow cytometer (BD Biosciences) within $1 \mathrm{~h}$.

Statistical analysis. One-way analysis of variance was used to determine statistical significance using Graphpad Prism 5.0 software (GraphPad Software, Inc., La Jolla, CA, USA). $\mathrm{P}<0.05$ and $\mathrm{P}<0.01$ were considered to indicate a statistically significant difference.

\section{Results}

Characterization of exosomes. The exosomes secreted by the NMuMG and 4T1 cells were isolated from serum-free culture supernatants using the exosome isolation kit as mentioned above. TEM was performed in order to observe the morphology of exosomes, and the size of the particles were determined from the images to be $\sim 100 \mathrm{~nm}$ (Fig. 1A). Western blot analysis was also performed to detect the exosomal marker proteins. The exosomal marker molecules CD63, CD81 and CD9 were detected in the exosome preparations derived from the NMuMG and 4T1 cell lines (Fig. 1B). The size distribution (Fig. 1C) and surface $\zeta$ potential (Fig. 1D) were also determined with the ZetaSizer Nano 
A

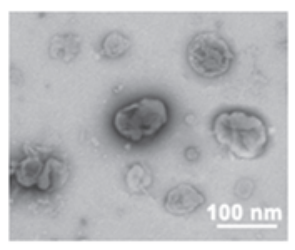

NMuMG-Exo

C
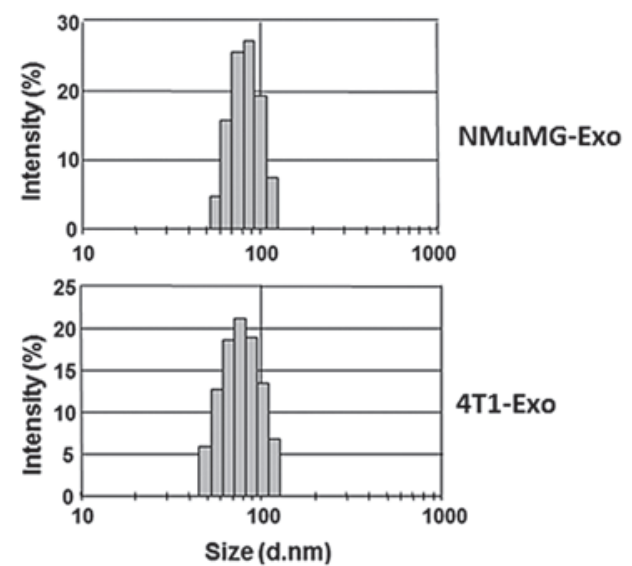

B

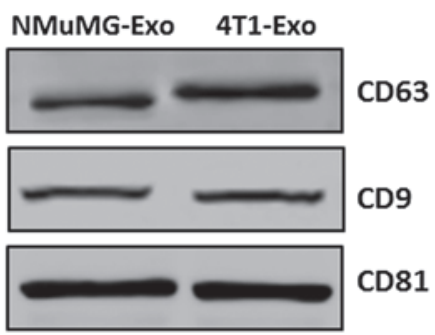

D
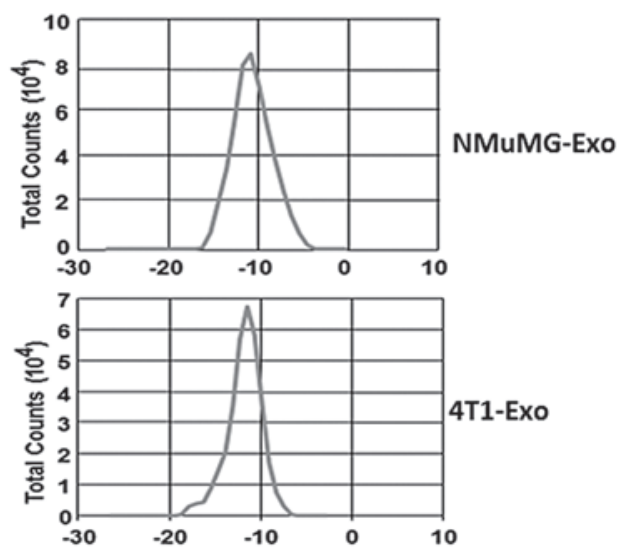

Figure 1. Characterization of exosomes purified from NMuMG and 4T1 cells. (A) The morphology of exosomes was imaged using electron microscopy and the diameter of particles were measured as $\sim 100 \mathrm{~nm}$. (B) Western blotting of exosomes for CD9, CD81 or CD63. (C) Size distribution and (D) surface $\zeta$ potential of exosomes secreted from cells.

A

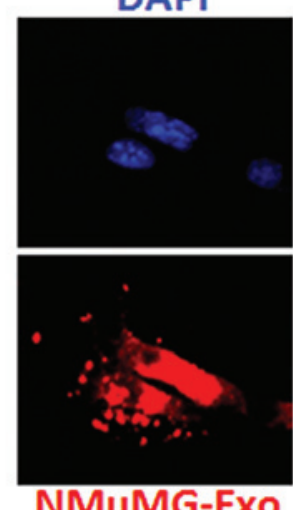

C

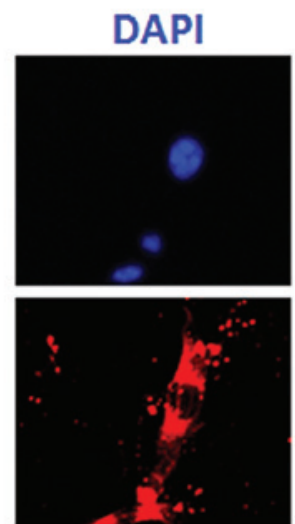

4T1-Exo
CD133

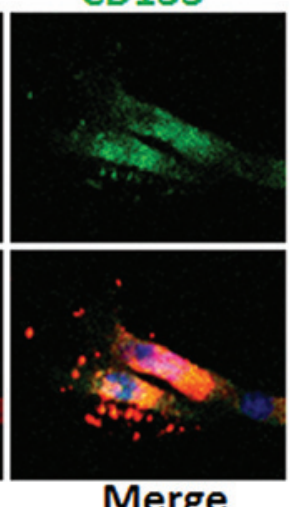

CD133
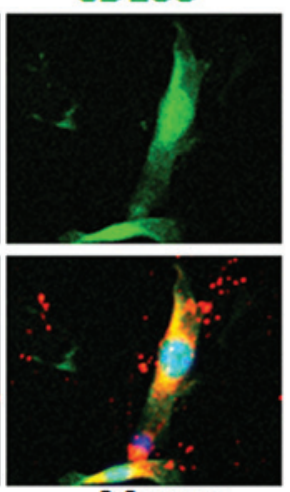

Merge
B

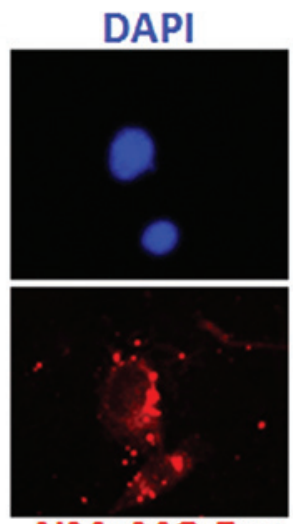

NMuMG-Exo

D
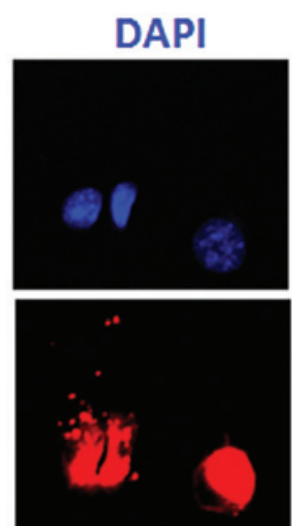

4T1-Exo
CD133

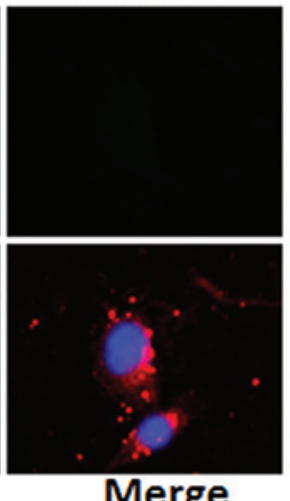

Merge

\section{CD133}
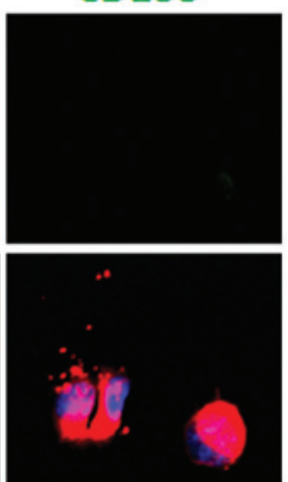

Merge

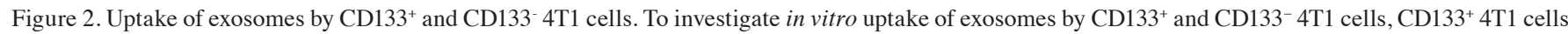
(green) were sorted by fluorescein isothiocyanate-anti-CD133 staining. The uptake was determined using confocal microscopy. Uptake of NMuMG-Exo by (A) $\mathrm{CD}^{3} 33^{+}$and (B) CD133- 4T1 cells. Uptake of 4T1-Exo by (C) CD133+ and (D) CD133- $4 \mathrm{~T} 1$ cells. 
A

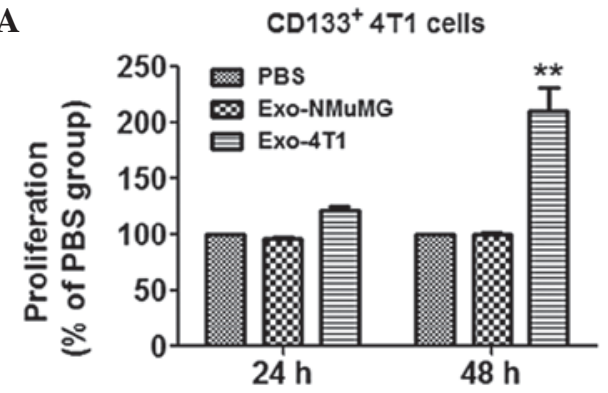

B

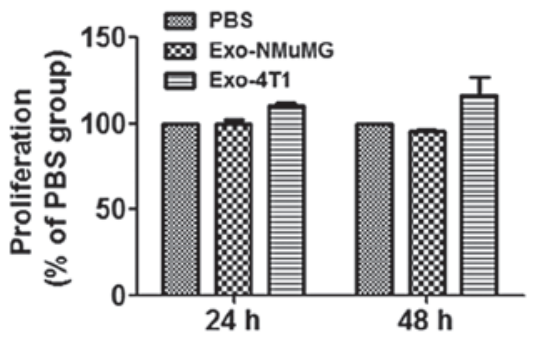

Figure 3. Proliferation of 4T1 cells. Proliferation of 4T1 cells following treatment with exosomes was assessed by ATPlite assay. Proliferation rate of (A) CD133 ${ }^{+}$ and (B) CD133- $4 \mathrm{~T} 1$ cells. ${ }^{* *} \mathrm{P}<0.01$ vs. PBS control.

A

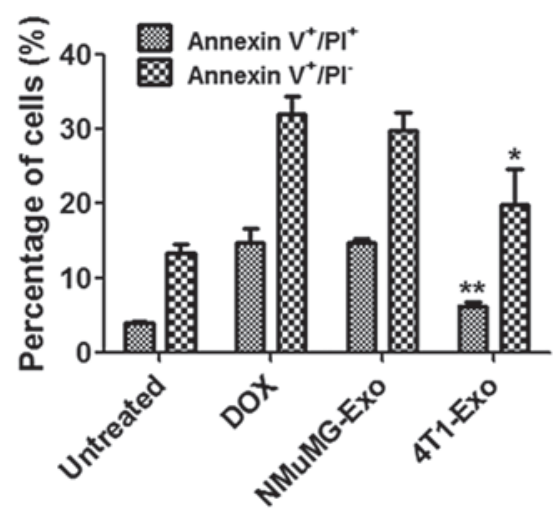

B

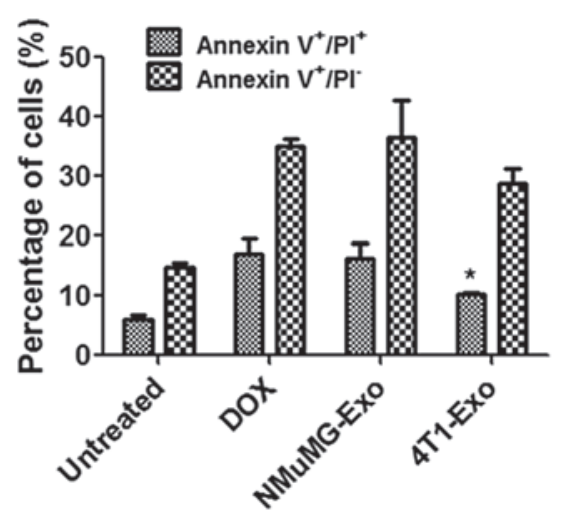

Figure 4. Apoptosis of 4T1 cells following exposure to doxorubicin. Apoptosis was quantified by fluorescence-activated cell sorting after Annexin V-fluorescein isothiocyanate and PI labeling. Statistical results of apoptosis of (A) CD133+ 4 T1 and (B) CD133- 4 T1 cells following treatment with doxorubicin ( ${ }^{*}<0.05$ and ${ }^{* *} \mathrm{P}<0.01$ vs. the untreated control). PI, propidium iodide.

ZS. Results indicated that the average sizes of the exosomes were $80.5 \mathrm{~nm}$ (NMuMG-Exo) and $89.6 \mathrm{~nm}$ (4T1-Exo) and the average $\zeta$ potentials were $-12.9 \mathrm{mV}$ (NMuMG-Exo) and $-15.8 \mathrm{mV}$ (4T1-Exo).

Exosomes from NMUMG or $4 T 1$ cells are internalized by the $\mathrm{CD}_{133^{+}}$and $\mathrm{CD} 133^{-} 4 \mathrm{T1}$ cells. NMuMG and 4T1 cell-derived exosomes were purified and characterized by size distribution, surface $\zeta$ potential and morphology using the TEM microscope. The uptake of exosomes by CD133 ${ }^{+}$ and CD133-4T1 cells was then examined. Exosomes were labeled with PKH26 as described in the methods. Next, $5 \mathrm{mg}$ exosomes were incubated with the $4 \mathrm{~T} 1$ cells. After $12 \mathrm{~h}$, the uptake of exosomes was imaged and the results indicated that the NMuMG (Fig. 2A and 2B) and 4T1 cell-derived exosomes (Fig. 2C and 2D) were taken up by the CD133 ${ }^{+}$and CD133-4T1 cells. These results suggest that exosomes from NMuMG or 4T1 cells may produce effects on CD133 positive and negative cells.

Exosomes from $4 T 1$ cells promote proliferation of $\mathrm{CD} 133^{+}$ 4 T1 cells. The microscope observations indicated that exosomes from NMuMG and 4T1 cells have the potential to be taken up by $\mathrm{CD}_{133^{+}}$and $\mathrm{CD} 133^{-}$cells, thus the effects of exosomes on proliferation of $\mathrm{CD} 133^{+}$and $\mathrm{CD} 133^{-}$cells were subsequently investigated by an ATPlite assay. Notably, exosomes from 4T1 cells significantly promoted the proliferation of $\mathrm{CD} 133^{+}$ cells after $48 \mathrm{~h}$ (Fig. 3A; ${ }^{* *} \mathrm{P}<0.01$ compared with control) but not CD133- cells (Fig. 3B).

Exosomes from $4 T 1$ cells inhibit apoptosis of $\mathrm{CD} 133^{+}$and CD133-4T1 cells. As presented in Fig. 3, it was demonstrated that exosomes from 4T1 cells significantly promoted the proliferation of $\mathrm{CD}_{133^{+}}$cells but had no effect on the proliferation of the CD133- cells. Therefore, the effect of exosomes on apoptosis of 4T1 cells was investigated. Following treatment with doxorubicin, Annexin V/PI staining was performed to quantify the apoptosis levels of 4T1 cells. The percentages of cells in each group were determined and presented as the mean \pm standard error. As shown in Fig. 4A, apoptosis of CD133+ $4 \mathrm{~T} 1$ cells was markedly suppressed by 4T1 cell-derived exosomes following treatment with doxorubicin (early apoptosis, ${ }^{*} \mathrm{P}<0.05$; late apoptosis, $\left.{ }^{* *} \mathrm{P}<0.01\right)$. In contrast to the proliferation data, the apoptosis of CD133-4T1 cells was also inhibited by 4T1 cell-derived exosomes to a certain extent (Fig. $4 \mathrm{~B} ;{ }^{*} \mathrm{P}<0.05$ ).

\section{Discussion}

Cell-to-cell communication is required for appropriate coordination among the different cell types in one organism. Cells may communicate with each other through soluble factors, through adhesion molecule-mediated cell-to-cell interactions, including cytonemes that connect neighboring cells, enabling ligand-receptor-mediated transfer of surface-associated 
molecules, or through tunneling nanotubules that establish conduits between cells, allowing the transfer of surface molecules and cytoplasmic components.

Tumor-derived exosomes exert antitumorigenic and protumorigenic effects by targeting different types of cells. Various studies have focused on the protumorigenic function (11) of tumor-derived exosomes, including their immunosuppressive properties (12), facilitation of tumor invasion and metastasis (13), and the promotion of tumor survival by transportation of RNA and protein (14). Diverse immunosuppressive effects of tumor-derived exosomes have been identified. Certain tumor cell lines are able to produce exosomes expressing death ligands, such as FasL and TRAIL, which can trigger the apoptotic death of activated T cells $(15,16)$. Additionally, Epstein-Barr virus-infected nasopharyngeal carcinoma has been demonstrated to release exosomes containing high levels of galectin-9, which induces apoptosis of mature Th1 lymphocytes subsequent to binding with the membrane receptor Tim-3 (17). Exosomes from murine-derived GL26 cells promote glioblastoma tumor growth by reducing the number and function of $\mathrm{CD}^{+} \mathrm{T}$ cells (18). Another study demonstrated that human melanoma- and colorectal carcinoma cell line-derived exosomes are also able to regulate monocyte differentiation into DCs toward the generation of myeloid-derived suppressor cells, and exert a TGF- $\beta 1$-mediated suppressive function on T cells in vitro (19). In addition to the suppression of the antitumor immunity, tumor-derived exosomes also contribute to the establishment of a premetastatic niche, generating a suitable microenvironment in metastatic sites by regulation of stromal cells, stimulating angiogenesis and remodeling the extracellular matrix (20). Protein and RNA, particularly miRNAs transported by exosomes to target cells, are another effective approach for cell-to-cell communication (21). There are few studies regarding the effects of tumor-derived exosomes on tumor stem cells. However, Cho et al (22) reported that exosomes from ovarian cancer cells induce adipose tissue-derived mesenchymal stem cells to acquire the physical and functional characteristics of tumor-supporting myofibroblasts, and that exosomes from breast cancer cells can convert adipose tissue-derived mesenchymal stem cells into myofibroblasts via a SMAD-mediated pathway. Myofibroblasts are a key source of matrix-remodeling proteins within the tumor microenvironment and thus participate in tumor angiogenesis.

To the best of our knowledge, the present study was the first to assess the function of $4 \mathrm{~T} 1$ mouse breast cancer cell-derived exosomes on $\mathrm{CD}_{133^{+}} 4 \mathrm{~T} 1$ cells in vitro. Uptake results indicated that exosomes from 4T1 cells were taken up by the $\mathrm{CD}_{133^{+}}$and CD133- $4 \mathrm{~T} 1$ cells. The in vitro proliferation assay demonstrated that the exosomes from 4T1 cells significantly enhanced proliferation of CD133+ but not CD133 4T1 cells. Also, the level of apoptosis in CD133+ $4 \mathrm{~T} 1$ cells following treatment with the apoptosis-inducing drug, doxorubicin, was significantly suppressed by 4T1-derived exosomes whilst CD133-4T1 cells were not. This phenotype suggests that tumor-derived exosomes may function as protumorigenic factors by promoting the proliferation and suppression of apoptosis of $\mathrm{CD} 133^{+}$tumor stem cells. The current study provides further understanding of the communication between tumor-derived exosomes and target cells.

\section{References}

1. Fan W, Tian XD, Huang E and Zhang JJ: Exosomes from CIITA-transfected CT26 cells enhance anti-tumor effects. Asian Pac J Cancer Prev 14: 987-991, 2013.

2. Wolfers J, Lozier A, Raposo G, et al: Tumor-derived exosomes are a source of shared tumor rejection antigens for CTL cross-priming. Nat Med 7: 297-303, 2001.

3. Clayton A, Mitchell JP, Court J, et al: Human tumor-derived exosomes selectively impair lymphocyte responses to interleukin-2. Cancer Res 67: 7458-7466, 2007.

4. Hartman ZC, Wei J, Glass OK, et al: Increasing vaccine potency through exosome antigen targeting. Vaccine 29: 9361-9367, 2011.

5. Clayton A, Al-Taei S, Webber J, et al: Cancer exosomes express CD39 and CD73, which suppress T cells through adenosine production. J Immunol 187: 676-683, 2011.

6. Cai Z, Yang F, Yu L, et al: Activated T cell exosomes promote tumor invasion via Fas signaling pathway. J Immunol 188: 5954-5961, 2012.

7. Munich S, Sobo-Vujanovic A, Buchser WJ, et al: Dendritic cell exosomes directly kill tumor cells and activate natural killer cells via TNF superfamily ligands. Oncoimmunology 1: 1074-1083, 2012.

8. Webber J, Steadman R, Mason MD, et al: Cancer exosomes trigger fibroblast to myofibroblast differentiation. Cancer Res 70: 9621-9630, 2010.

9. Bonito MD, Cantile M, Malzone G, et al: The prognostic role of cancer stem cells in breast tumors. J Clin Med Res 5: 325-326, 2013.

10. Wang Q, Zhuang X, Mu J, et al: Delivery of therapeutic agents by nanoparticles made of grapefruit-derived lipids. Nat Commun 4: 1867, 2013

11. Yang C and Robbins PD: The roles of tumor-derived exosomes in cancer pathogenesis. Clin Dev Immunol 2011: 842849, 2011.

12. Yang $C$ and Robbins PD: Immunosuppressive exosomes: a new approach for treating arthritis. Int J Rheumatol 2012: 573528, 2012.

13. Stoeck A, Keller S, Riedle S, et al: A role for exosomes in the constitutive and stimulus-induced ectodomain cleavage of L1 and CD44. Biochem J 393: 609-618, 2006.

14. Zomer A, Vendrig T, Hopmans ES, et al: Exosomes: Fit to deliver small RNA. Commun Integr Biol 3: 447-450, 2010.

15. Andreola G, Rivoltini L, Castelli C, et al: Induction of lymphocyte apoptosis by tumor cell secretion of FasL-bearing microvesicles. J Exp Med 195: 1303-1316, 2002.

16. Huber V, Fais S, Iero M, et al: Human colorectal cancer cells induce T-cell death through release of proapoptotic microvesicles: role in immune escape. Gastroenterology 128: 1796-1804, 2005.

17. Keryer-Bibens C, Pioche-Durieu C, Villemant C, et al: Exosomes released by EBV-infected nasopharyngeal carcinoma cells convey the viral latent membrane protein 1 and the immunomodulatory protein galectin 9. BMC Cancer 6: 283, 2006.

18. Liu ZM, Wang YB and Yuan XH: Exosomes from murine-derived GL26 cells promote glioblastoma tumor growth by reducing number and function of $\mathrm{CD}^{+} \mathrm{T}$ cells. Asian Pac J Cancer Prev 14: 309-314, 2013.

19. Cai Z, Zhang W, Yang F, et al: Immunosuppressive exosomes from TGF- $\beta 1$ gene modified dendritic cells attenuate Th17-mediated inflammatory autoimmune disease by inducing regulatory $\mathrm{T}$ cells. Cell Res 22: 607-610, 2012.

20. Peinado H, Lavotshkin S and Lyden D: The secreted factors responsible for pre-metastatic niche formation: old sayings and new thoughts. Semin Cancer Biol 21: 139-146, 2011.

21. Channavajjhala SK, Rossato M, Morandini F, et al: Optimizing the purification and analysis of miRNAs from urinary exosomes. Clin Chem Lab Med 52: 345-354, 2014.

22. Cho JA, Park H, Lim EH, et al: Exosomes from ovarian cancer cells induce adipose tissue-derived mesenchymal stem cells to acquire the physical and functional characteristics of tumor-supporting myofibroblasts. Gynecol Oncol 123: 379-386, 2011. 